\title{
SAÚDE OCULAR EM ESCOLARES E A PRÁTICA DOS ENFERMEIROS DA ATENÇÃO BÁSICA*
}

\author{
Raquel Malta Fontenele ${ }^{1}$, Ana Inês Sousa ${ }^{2}$, Alexandra Schmitt Rasche ${ }^{2}$
}

\begin{abstract}
RESUMO: Objetivou-se descrever o perfil e as práticas dos enfermeiros da Equipe de Saúde na Família voltadas para saúde ocular em escolares. Trata-se de estudo descritivo, em uma Área de Planejamento do município do Rio de Janeiro, no período de maio a junho de 2013. Dentre as ações destaca-se o quantitativo $62(66 \%)$ dos enfermeiros que identificaram acuidade visual prejudicada durante consulta de enfermagem e os que afirmaram $17(18,08 \%)$ ter realizado ação de saúde nas escolas. Evidencia-se que todos os entrevistados pontuaram a necessidade de receber informações sobre o teste de acuidade visual. As ações voltadas para saúde ocular de escolares precisam ser aprimoradas, ressaltando-se a ampliação da atuação do enfermeiro para ações no âmbito da comunidade escolar. Recomenda-se atualização periódica sobre o tema e incentivo à adoção de práticas rotineiras nas ações de prevenção e promoção de saúde nas escolas, favorecendo melhorias na qualidade da assistência ofertada.
\end{abstract}

DESCRITORES: Serviços de Enfermagem Escolar; Saúde Ocular; Enfermagem em Saúde Pública.

\section{OCULAR HEALTH IN SCHOOLCHILDREN AND THE NURSES' PRACTICE IN PRIMARY HEALTHCARE}

\begin{abstract}
The aim was to describe the profile and the practices of the nurses of the Family Health Team geared towards ocular health in schoolchildren. It is a descriptive study, in a Planning Area (PA) of the municipality of Rio de Janeiro, undertaken in May - June 2013. Among the actions, emphasis is placed on the number - $62(66 \%)$ - of the nurses who identified impaired visual acuity during the nursing consultation, and those who asserted - $17(18.08 \%)$ - that they had undertaken health actions in the schools. It is evidenced that all of those interviewed indicated the need to receive information on the visual acuity test. The actions directed towards schoolchildren's ocular health need to be improved, with emphasis placed on extending the nurse's work to include actions in the ambit of the school community. Periodical updating on the issue is recommended, along with encouragement to adopt routine practices in the health promotion and prevention actions undertaken in the schools, favoring improvements in the quality of the care offered.
\end{abstract}

DESCRIPTORS: School Nursing; Eye health; Public Health Nursing.

\section{SALUD OCULAR EN ESCOLARES Y LA PRÁCTICA DE ENFERMEROS DE ATENCIÓN BÁSICA}

RESUMEN: Se objetivó describir el perfil y las prácticas de enfermeros del Equipo de Salud de la Familia orientadas a salud ocular en escolares. Estudio descriptivo, en un Área de Planificación del Municipio de Río de Janeiro, entre mayo y junio de 2013. Entre las acciones, destaca la cantidad de $62(66 \%)$ de enfermeros que identificaron agudeza visual disminuida durante consulta de enfermería y los 17 (18,08\%) que afirmaron haber realizado campañas de salud en escuelas. Resulta evidente que todos los entrevistados puntualizaron necesidad de recibir capacitación sobre test de agudeza visual. Las campañas orientadas a la salud ocular en estudiantes necesitan mejoras, resaltándose ampliación de actuación del enfermero en campañas en el marco de la comunidad escolar. Se recomienda actualización periódica sobre el tema e incentivación de adopción de prácticas de rutina en las campañas de prevención y promoción de salud en escuelas, permitiendo mejorar la calidad de atención ofrecida.

DESCRIPTORES: Servicios de Enfermería Escolar; Salud Ocular; Enfermería en Salud Pública.

\footnotetext{
*Artigo extraído da dissertação de Raquel Malta Fontenele, intitulada: “Saúde ocular em escolares: conhecimentos, atitudes e práticas dos enfermeiros". Escola de Enfermagem Anna Nery-UFRJ, 2013.

${ }^{1}$ Enfermeira. Doutoranda em Enfermagem. Escola de Enfermagem Anna Nery Universidade Federal do Rio de Janeiro. Rio de Janeiro, RJ, Brasil.

${ }^{2}$ Enfermeira. Doutora em Enfermagem. Docente da Escola de Enfermagem Anna Nery Universidade Federal do Rio de Janeiro. Rio de Janeiro, RJ, Brasil.
} 


\section{- INTRODUÇÃO}

Dentre os deficientes visuais, 148 mil pessoas são cegas, sendo que de acordo com as estimativas, cerca de $90 \%$ dos casos poderiam ter sido evitados com ações de promoção da saúde e prevenção e detecção precoce. A prevalência de cegueira infantil, no Brasil, é de 01 a 1,5 para cada 1.000 crianças e aproximadamente $20 \%$ dos escolares brasileiros apresentam alguma alteração ocular ${ }^{(1-2)}$.

A prevenção e a detecção precoce de deficiências oculares na infância são os melhores recursos para evitar possíveis consequências de alterações na acuidade visual e essas ações poderão ser desenvolvidas, preferencialmente, no ambiente escolar, por se tratar de instituições com grande concentração de crianças, atingindo um maior número possível ${ }^{(3)}$. A base do Programa de Saúde na Escola (PSE)(1) é a articulação entre a Escola e a Rede Básica de Saúde, o que implica intersetorialidade compartilhada dos setores saúde e educação com as demais redes sociais para o desenvolvimento do PSE, obtendo mais do que uma oferta de serviços num mesmo território, propicia a sustentabilidade das ações a partir da conformação das redes de corresponsabilidade. Os programas nacionais conduzem e orientam a adesão municipal e a pactuação das metas, assim como apontam para a necessidade de capacitar e subsidiar os profissionais da atenção básica na prática da promoção da saúde escolar, em especial a ações de saúde ocular, nesta faixa etária que demonstra fragilidade e assim merece atenção redobrada ${ }^{(1)}$.

A prevenção, a promoção de saúde, o diagnóstico precoce e o controle da visão devem ser planejados e executados no objetivo de reduzir os casos e as consequências das alterações visuais nas crianças. O enfermeiro tem papel fundamental nesse processo, pois além de ter a oportunidade de ter contato com as crianças em suas diferentes fases de desenvolvimento, poderá realizar orientação com os familiares e cuidadores para identificar qualquer sinal de acuidade visual alterada e assim procurar por um profissional da saúde especializado ${ }^{(1,4)}$.

A inserção do enfermeiro no ambiente escolar, como profissional que promove o cuidado, é fundamental e pode gerar efeitos positivos nas escolhas de saúde dos indivíduos que utilizam o ambiente escolar. Evidências internacionais indicam que enfermeiros que trabalham nos cuidados primários podem fornecer cuidados eficazes e alcançar resultados positivos para a saúde dos usuários, devido a sua habilidade, conhecimento e receptividade, notando a importância do enfermeiro frente à Equipe de Saúde da Família no intuito de melhorar a qualidade da assistência primáriaa ${ }^{(5-6)}$.

Entretanto, justifica-se a temática deste artigo na evidência da participação de profissionais da atenção básica atuando na promoção da saúde ocular em crianças em fase escolar, no escopo de auxiliar na identificação precoce, sendo essenciais para o controle da deficiência visual na infância.

Busca-se despertar atenção sobre a importância do enfermeiro em realizar ações de intersetorialidade dentro da Estratégia de Saúde da Família (ESF), em conjunto com os demais profissionais da equipe. Dessa forma, tem-se a intenção de enaltecer a prática de promoção da saúde voltada para a saúde ocular de escolares desenvolvidas pelos enfermeiros e auxiliar gestores no que diz respeito ao incentivo das práticas e propiciar ferramentas para a realização das ações de saúde ocular. Portanto, não se está em busca de avaliar erros ou denunciar, porém espera-se contribuir com o conhecimento científico e evidenciar os pressupostos da Política Nacional de Atenção Básica vigente, dentre eles, estreitar o elo entre as áreas de saúde e educação, associadas à promoção de saúde com qualidade e eficácia.

Assim este estudo tem como propósito descrever o perfil e as práticas dos enfermeiros da Equipe de Saúde na Família voltadas para saúde ocular em escolares, em uma Área de Planejamento do município do Rio de Janeiro.

\section{- MÉTODO}

Para o desenvolvimento da pesquisa adotou-se o estudo descritivo, realizado na Área de Planejamento (AP) 3.1 do município do Rio de Janeiro, no período de maio a junho de 2013. O cenário, AP 3.1, foi escolhido pela quantidade total de equipes ESF completas ao comparada com outras áreas do município, dado este obtido na Secretaria Municipal de Saúde, junto com o número do Cadastro 
Nacional de Estabelecimento de Saúde de cada profissional enfermeiro da ESF da área selecionada.

A população de referência foi constituída por todos os 119 enfermeiros cadastrados na ESF no período de coleta de dados, independente do seu tipo de vínculo (contrato ou estatutário), lotados na área supracitada. Do total de enfermeiros, 19 foram considerados inelegíveis por se enquadrarem nos critérios de exclusão, ou seja, tinham menos de três meses de atuação naquela Unidade de Saúde, estavam em férias ou licença maternidade e/ou eram residentes. Da população elegível para o estudo, houve seis perdas por incompatibilidade de agenda com os profissionais, mesmo após três tentativas.

Excluídas as perdas, a população do estudo que atendeu aos critérios de inclusão, ou seja, ser enfermeiro regularmente contratado ou estatutário e com experiência de atuação na ESF, constituiu-se de94 enfermeiros. Ressalta-se que o Termo de Consentimento Livre e Esclarecido (TCLE) foi apresentado, lido em voz alta, esclarecidas as dúvidas e assinado pelas partes (pesquisador e participante).

A coleta de dados foi realizada por meio de um questionário para levantamento das variáveis: sexo, idade, titulação, tempo de formação, tempo de atuação na ESF. Com relação às ações de saúde ocular em escolares, foi questionado se o enfermeiro já havia identificado algum caso de alteração visual durante a realização da consulta de enfermagem, durante suas atividades desenvolvidas na atenção básica, e o que o levou a investigar, podendo ser queixa, encaminhamento, indicação ou durante a realização do exame físico. Outro dado levantado foi o interesse em receber informações sobre a temática de saúde ocular, sob a ótica do enfermeiro, e qual a melhor forma de repassar essas informações.

Foi realizada análise descritiva exploratória dos dados, apresentada através de gráficos e tabelas de frequência absoluta. O programa estatístico utilizado foi Epi-Info (versão 3.5.2) e as perguntas que possibilitavam a marcação de mais de uma opção de resposta tinham sua análise feita através da opção Check Box do programa estatístico utilizado. O estudo foi aprovado pelo Comitê de Ética e Pesquisa da Escola de Enfermagem Anna Nery da Universidade Federal do Rio de Janeiro, sob o número de CAAE 12455513.4.0000.5238 e de protocolo 240.019, aprovado em 08 de abril de 2013.

\section{RESULTADOS}

Dentre os resultados, vale uma breve discussão sobre o perfil dos enfermeiros inseridos na ESF, que se apresenta na Tabela 1. Dentre os dados, destaca-se a predominância feminina (84\%), na faixa etária entre os 20 aos 29 nos (43,6\%) seguidos da faixa etária dos 30 aos 39 anos (39,4\%). Com relação às características profissionais, metade da população estudada tinha entre um e cinco anos de formação acadêmica (49,9\%), sendo que 72 (76,6\%) enfermeiros disseram ter concluído o curso de especialização. O tempo de serviço na unidade em que estavam lotados, no momento da coleta de dados, teve como evidencia de um até dois anos de atuação $(35,1 \%)$.

Dos enfermeiros que responderam positivamente (66\%) com relação à identificação de alteração visual durante a consulta de enfermagem (atividade estabelecida pela atenção básica), ao serem questionados sobre o motivo, 35 (56,4\%) enfermeiros afirmaram que investigaram tal caso por conta da queixa do acompanhante ou familiar de dificuldade aparente de enxergar, seguido da queixa do escolar que no ponto de ônibus, não conseguia enxergar o número de identificação de longe. Esses resultados são descritos na Tabela 2 .

Dentre os que responderam 'outros', alguns participantes relataram identificação de possível alteração durante a "anamnese" e um relatou que recebeu um bilhete do educador sobre possível sintoma de alteração visual, destacando, mesmo que de forma inicial e não assídua, a relação entre profissionais de saúde e educação. Vale destacar que 4 participantes $(6,45 \%)$ pontuaram ter recebido alguma notificação do profissional de educação para algum sinal ou sintoma de alteração visual na criança, o que nos remete a uma ligação ente a ESF e a unidade escolar.

Ainda com relação à prática dos enfermeiros, foi questionado se já havia realizado alguma ação de saúde ocular nas escolas em sua trajetória profissional na ESF, independente se na área de atuação; 77 $(81,9 \%)$ disseram que não realizaram ações de saúde ocular nas escolas (Tabela 3 ).

Ao serem questionados se gostariam de receber mais informações sobre acuidade visual, todos os 
enfermeiros (100\%) entrevistados responderam positivamente e se mostraram interessados sobre o tema. Logo em seguida, foi questionada a forma como melhor receberiam essas informações, sendo que cursos e oficinas foram as maneiras mais pontuadas pelos participantes do estudo (Tabela 4).

Tabela 1 - Distribuição da população segundo características sócio demográficas e formação profissional. Rio de Janeiro, RJ, Brasil, 2013

\begin{tabular}{lcc}
\hline Variáveis & $\mathbf{n}$ & $\mathbf{\%}$ \\
\hline Sexo & & \\
\hline Feminino & 79 & 84 \\
\hline Masculino & 15 & 16 \\
\hline Faixa etária (em anos) & & \\
\hline 20 a 29 & 41 & 43,6 \\
\hline 30 a 39 & 37 & 39,4 \\
\hline 40 a 49 & 12 & 12,8 \\
\hline 50 a 60 & 4 & 4,3 \\
\hline Tempo de Formação & & \\
\hline Até onze meses & 1 & 1,1 \\
\hline Entre 1 e 5 anos & 47 & 49,9 \\
\hline Mais de 5 anos & 31 & 33 \\
\hline Mais de 10 anos & 15 & 16 \\
\hline Titulação & & \\
\hline Graduação & 13 & 13,8 \\
\hline Especialização & 72 & 76,6 \\
\hline Mestrado & 7 & 7,4 \\
\hline Não Informado & 2 & 2,1 \\
\hline Tempo de Atuação na ESF na unidade & & \\
\hline Até onze meses & 4 & 4,3 \\
\hline 1 ano & 33 & 35,1 \\
\hline 2 anos & 24 & 25,5 \\
\hline 3 anos & 17 & 18,1 \\
\hline 4 a 10 anos & 14 & 14,9 \\
\hline Não informado & 2 & 2,1 \\
\hline TOTAL & 94 & 100
\end{tabular}

Tabela 2-Distribuição da população, segundo o motivo para investigar a alteração visual durante a consulta de enfermagem. Rio de Janeiro, RJ, Brasil, 2013

\begin{tabular}{|c|c|c|}
\hline $\begin{array}{l}\text { Motivos para investigar acuidade visual } \\
\text { durante a consulta de enfermagem }\end{array}$ & $n=62$ & $\%$ \\
\hline $\begin{array}{l}\text { Queixa do acompanhante, ou } \\
\text { familiar, de alguma dificuldade } \\
\text { aparente de enxergar }\end{array}$ & 35 & 56,4 \\
\hline Queixa do Escolar & 24 & 38,7 \\
\hline $\begin{array}{l}\text { Indicação de um profissional de } \\
\text { educação }\end{array}$ & 4 & 6,4 \\
\hline $\begin{array}{l}\text { Indicação de um profissional de } \\
\text { saúde }\end{array}$ & 3 & 4,8 \\
\hline $\begin{array}{l}\text { Identificado no momento do exame } \\
\text { físico }\end{array}$ & 19 & 30,6 \\
\hline Outros & 4 & 6,4 \\
\hline
\end{tabular}

Obs.: Percentual calculado com base nos entrevistados que afirmaram já ter identificado alteração visual $(n=62)$.

Obs. 2: A pergunta que gerou este quadro possibilitava a marcação de mais de uma opção de resposta.

Tabela 3 - Distribuição da população, segundo a realização de alguma ação de saúde ocular nas escolas. Rio de Janeiro, RJ, Brasil, 2013

\begin{tabular}{|ccc|}
$\begin{array}{l}\text { Realização de ação de saúde ocular nas } \\
\text { escolas }\end{array}$ & $\mathbf{n}$ & $\%$ \\
\hline Sim & 17 & 18,08 \\
\hline Não & 77 & 81,91 \\
\hline Total & 94 & 100 \\
\hline
\end{tabular}

Tabela 4 - Distribuição da população, segundo a melhor forma de receber informação sobre o tema acuidade visual. Rio de Janeiro, RJ, Brasil, 2013

\begin{tabular}{lcc}
$\begin{array}{l}\text { Formas para receber informações } \\
\text { sobre o tema acuidade visual }\end{array}$ & $\mathbf{n = 9 4}$ & $\%$ \\
\hline Cursos e Oficinas & 66 & 70,2 \\
\hline Aulas de educação continuada & 56 & 59,6 \\
\hline Informativos impressos & 18 & 19,1 \\
\hline $\begin{array}{l}\text { Meios eletrônicos } \\
\text { Obs.: Percentual calculado com base no total de entrevistados } \\
\text { (n=94). }\end{array}$ \\
$\begin{array}{l}\text { Obs. 2: A pergunta que gerou esta tabela possibilitava a } \\
\text { marcação de mais de uma opção de resposta. }\end{array}$
\end{tabular}




\section{DISCUSSÃO}

O objetivo deste artigo de descrever o perfil e as práticas dos enfermeiros inseridos na ESF voltadas para a saúde ocular de escolares justifica-se pela intenção de conhecê-los e assim, traçar a melhor estratégia de incentivo para as práticas de atenção integral (prevenção, promoção e atenção) à saúde de estudantes da educação básica pública brasileira.

A ressignificação do trabalho do enfermeiro no âmbito da atenção básica foi desencadeada pelo processo de implantação do Sistema Único de Saúde (SUS), buscando atender aos seus princípios e pelo conceito de saúde ampliado ao contexto social ${ }^{(7)}$. Entretanto, o enfermeiro ficou responsável não somente pela organização do serviço como também pelo processo de planejamento e implementação de ações e avaliações clínicas na unidade de saúde e na relação dela com os ambientes do território. Portanto, conhecer os enfermeiros que atuam na atenção básica fornece dados para entendimento das problemáticas de recursos humanos, além de estimular a participação no processo de planejamento e implementação de ações no território, atendendo à carga horária da carta de serviços pactuada e contribuindo no planejamento de temas para capacitação dos profissionais.

Para discussão neste estudo, destaca-se que os enfermeiros da área estudada tem a predominância do sexo feminino, resultados semelhantes foram apresentados em outros estudos de regiões diferentes do país ${ }^{(8-9)}$. Tal fato reproduz a característica histórica da enfermagem, profissão exercida em maior número por mulheres. Com relação à faixa etária, o estudo realizado em Cuiabá(9) obteve resultado semelhante, onde a maioria dos participantes se encontrava entre a faixa dos 26 aos 30 anos, corroborando com encontrado no município do Rio de Janeiro, ou seja, predomina na atenção básica o jovem enfermeiro.

Evidencia-se o grande número de participantes que possuem um curso de especialização, resultado semelhante foi discutido em estudos, em que se aponta o investimento em mais formação, tanto com incentivo do sistema de saúde vigente, quanto pela busca pessoal de conhecimentos específicos da atenção primária ${ }^{(8-9)}$. Espera-se, ao investigar o tempo de atuação do profissional, que quanto maior o tempo de atuação na atenção básica, maiores as possibilidades de vivenciar experiências na profissão, assim como o estabelecimento de vínculos efetivos entre a equipe e os usuários.

A permanência de profissionais em uma organização está relacionada além da estabilidade adquirida pelo tempo de serviço e pelo reconhecimento profissional, também ao envolvimento deste profissional com os serviços prestados e sua identificação com estes modelos. Assim, uma "alta rotatividade" de profissionais é discutida quando os profissionais apontam que a saída de um membro da equipe acarreta sentimentos negativos quanto ao desempenho do grupo. Surgem alguns questionamentos, como por exemplo, a qualidade da assistência prestada que em geral passa a ser entendida como diminuída ${ }^{(10)}$.

Ao aprofundar o conhecimento e a prática vivenciados na profissão, é possível ao enfermeiro da atenção básica ampliar a sua visão de atuação em seu território, o que leva a identificar a escola como área de importante ação, por ser um local de formação de senso crítico e moral, em que hábitos básicos de vida são moldados para escolares e comunidade escolar e, principalmente, por ser local fértil para o desenvolvimento de ações de educação, prevenção e promoção da saúde ${ }^{(11-12)}$.

Ao discutir a prática de ações de saúde ocular, vale destacar a importância de se detectar os problemas visuais na criança ainda que em idade pré-escolar e escolar. Essa pontuação torna-se importante pelo fato de que é nesta faixa etária que ocorre o pleno desenvolvimento do aparelho visual, onde triagens e identificação de alteração visual resultam em maior resolução de problemas e suas consequências poderiam ser atenuadas ou mesmo evitadas ${ }^{(4)}$.

Corroborando com a discussão, com relação às ações de saúde de crianças em fase escolar, ressaltase que o perfil de morbidade no Brasil dos adolescentes revela a presença de doenças crônicas, transtornos psicossociais, fármaco-dependência, doenças sexualmente transmissíveis e outros agravos. Essas características de perfil de saúde exigem da Equipe de Saúde da Família planejamento das ações a fim de reduzir os agravos de saúde, pactuando ações que envolvam outros setores da sociedade, como a escola e a comunidade adscrita e principalmente os familiares, para que possam ser garantidas 
melhores condições de saúde, favorecendo a interação ESF e escolas para promover saúde e melhorar a qualidade da assistência ${ }^{(13)}$.

Relacionado à temática deste estudo, o exame da acuidade visual é uma triagem importante ao longo do desenvolvimento da criança e é a equipe da ESF responsável ou possível protagonista na detecção de doenças oculares óbvias e também daquelas assintomáticas e de curso insidioso ${ }^{(14)}$. Um teste de acuidade visual dinâmico pode desempenhar um papel importante e representa elementochave no desenvolvimento social e cognitivo relacionado à idade do escolar de forma a garantir adequada e oportuna avaliação e proporcionando intervenção precoce de especialistas ${ }^{(15)}$. Ressalta-se que a técnica mais utilizada é de baixo custo e consiste em realizar uma triagem no ambiente escolar em que o teste da acuidade visual é aplicado, com a Tabela Optométrica Decimal de Snellen ${ }^{(14)}$ forma mais simples de diagnosticar a limitação da visão.

Apontamos as evidências do pouco desenvolvimento de práticas voltadas para saúde ocular encontradas nos resultados deste estudo, também comparadas às avaliações auditivas e oftalmológicas realizadas quando se levantam os dados advindos do Programa Nacional de Melhoria do Acesso e Qualidade da Atenção Básica, que pontuou apenas $27 \%$ e 17\%, respectivamente, dos profissionais que atuam na atenção básica realizam ou planejam essas ações, o que ressalta o alerta para a necessidade de melhoria neste quadro(16).

A relação da atenção básica com o ambiente escolar constitui-se um ambiente fértil para formação de futuros cidadãos. Para os profissionais que atuam na ESF, corresponde ampliar o alcance e o impacto das práticas voltadas para a saúde do escolar e aumentar a abrangências de programas como o PSE, quanto às políticas de atenção básica voltadas para melhoria da qualidade da assistência, ambas relativas aos escolares e suas famílias, otimizando a utilização dos espaços para efetivação das ações ${ }^{(1,14)}$.

No que diz respeito à necessidade de receber mais informações, ficou evidente a importância de melhorar e oferecer subsídios teóricos para os profissionais da atenção básica realizarem e pactuarem as ações com efetividade. Portanto, o Guia de Sugestões de Atividades para a Semana Saúde da Escolar ${ }^{(1)}$ poderá ser uma ferramenta para auxiliar neste processo de capacitação e para aplicação da técnica da triagem de acuidade visual, além de outros materiais como o Caderno da Atenção Básica de Saúde na Escola $^{(1,14)}$. Nestes materiais são explicados, de forma simples, os pontos para a realização do exame, tais como: o domínio da técnica de aplicação, o preparo do material, a escolha e o preparo do local, o treinamento do auxiliar, o preparo dos escolares, o preparo do material para o registro técnico da triagem e o reteste.

Abrindo para uma discussão de ações em outros países, como na Noruega, o enfermeiro tem uma relação importante com o ambiente escolar. As enfermeiras realizam visitas domiciliares para realizar a vacinação e acompanham o desenvolvimento das crianças, assim como também aconselham e orientam a comunidade sobre os serviços em clínicas de saúde da criança no território. Um estudo realizado na Noruega em 2013 apontou um quantitativo de 2.069 enfermeiras atuantes em saúde da família, prestando serviços em clínicas e serviços de saúde escolar ${ }^{(17)}$.

Na realidade nacional, até 2012 o Programa de Saúde na Escola Nacional somente era articulado às equipes de ESF. Em 2013 o programa universaliza suas ações e seu protocolo de adesão, aumentado pelo o número de municípios pactuados, e passa a vincular suas ações à atenção básica, em que as ações são programadas junto à escola do território pelo menos uma vez ao mês ${ }^{(1)}$. No entanto, a existência ou não das Equipes Especiais, que na realidade não são preconizadas pelo SUS, surgiram de uma ação autônoma de alguns municípios, que montaram essas equipes para atenderem somente as escolas PSE, tornando evidente e passível de discussão e avaliação a responsabilidade da equipe de saúde da família com as ações de promoção de saúde escolar.

\section{- CONCLUSÃO}

Facilitar o acesso de crianças e adolescentes ao sistema de garantia de direitos sociais, como saúde, educação e desenvolvimento social, corresponde às metas do PSE, assim como fortalecer as ações integradas de promoção de saúde e proteção social no território adscrito. No entanto, o profissional 
enfermeiro precisa de incentivo para ampliar e solidificar sua atuação nas ações de saúde escolar através de capacitações, inclusive nos testes de acuidade visual, de forma periódica ou no mínimo anualmente.

Considerada como uma boa conduta de prática de saúde, pesquisar a acuidade visual em escolares, tanto no ambiente escolar como também durante a consulta de enfermagem, constitui uma ação do enfermeiro em conjunto com a equipe de saúde e educação inserida no território. Com uma identificação precoce e uma rápida intervenção, espera-se auxiliar no processo de aprendizagem do escolar, obtendo melhorias que irão subsidiar o desenvolvimento da qualidade de vida das crianças.

O estudo apontou que todos os enfermeiros apresentaram uma atitude favorável no que diz respeito à mudança de rotina, demonstrando interesse em participar de cursos e capacitações, o que confirma o empenho em aderir à prática voltada para saúde ocular. Assim, recomendam-se adicionar na agenda mensal e tornar rotina algumas horas destinadas para promoção da saúde em conjunto com os demais profissionais da equipe de saúde da família, atendendo ao modelo multiprofissional nas ações de saúde na escola.

Assim destacamos a necessidade de realização de novas pesquisas com ênfase no processo de trabalho do enfermeiro no âmbito da atenção básica, com o objetivo de fortalecer e enaltecer os serviços de enfermagem escolar, gerando informações e conhecimento e também contemplando as atividades de educação permanente no âmbito da saúde pública.

\section{- REFERÊNCIAS}

1. Ministério da Saúde/ Ministério da Educação (BR). Programa Saúde na Escola. Manual Instrutivo Programa Saúde na Escola 2013. [Internet] 2013 [acesso em 10 set 2013]. Disponível: http://www.pmf.sc.gov.br/arquivos/ arquivos/pdf/19_06_2013_16.21.18.880166244cb983df2c85e0bcc746a73b.pdf.

2. Cezario KG, Oliveira PMP, Baptista RS, Pinheiro AKB, Pagliuca LMF. Promoção da saúde e deficiência visual: produção das pós-graduações brasileiras. Rev Rene [Internet]. 2010; 11(2) [acesso em 20 set 2013]. Disponível: http://www.revistarene.ufc.br/vol11n2_pdf/a21v11n2.pdf.

3. Rodrigues Junior JC, Dantas RA, Alves RS, Rebouças CB, Leite IF. Visual acuity and its implications for school performance. Rev enferm UFPE on line. [Internet] 2012; 6(11) [acesso em 23 jul 2014]. Disponível: http://www. revista.ufpe.br/revistaenfermagem/index.php/revista/article/view/3279.

4. Laignier MR, Castro MA, Sá PSC. De olhos bem abertos: investigando acuidade visual em alunos de uma escola municipal de Vitória. Esc. Anna Nery. [Internet] 2010; 14(1) [acesso em 20 set 2014]. Disponível: http://dx.doi. org/10.1590/S1414-81452010000100017.

5. Balbino CM, Silvino ZR. A gerência do cuidado de enfermagem na comunidade escolar: estudo de caso. Online braz j nurs. [Internet] 2012; 11(2) [acesso em 14 abr 2014]. Disponível: http://dx.doi.org/10.5935/16764285.2012S032.

6. Parker R, Forrest L, Ward N, McCracken J, Cox D, Derrett J. How acceptable are primary health care nurse practitioners to Australian consumers?. Collegian. 2013;20(1):35-41.

7. Matumoto S, Fortuna CM, Kawata LS Mishima SM, Pereira MJB. Nurses' clinical practice in primary care: a process under construction. Rev. Latino-Am. Enfermagem. [Internet] 2011; 19(1) [acesso em 14 abr 2014]. Disponível: http://dx.doi.org/10.1590/S0104-11692011000100017.

8. Silva VG, Motta MCS, Zeitoune RCG. A prática do enfermeiro na Estratégia Saúde da Família: o caso do município de Vitória/ES. Rev. Eletr. Enf. [Internet] 2010; 12(3) [acesso em 23 jul 2014]. Disponível: http://dx.doi. org/10.5216/ree.v12i3.5278.

9. Corrêa ACP, Araújo EF, Ribeiro AC, Pedrosa ICF. Sociodemographic and professional profile of primary health care nurses in Cuiabá - Mato Grosso. Rev. Eletr. Enfer. [Internet] 2012; 14(1) [acesso em 14 abr 2015]. Disponível: http://www.revistas.ufg.br/index.php/fen/article/view/12491.

10. Tironi NM, Silva LGC, Dellaroza MSG, Haddad MCL, Vannuchi MTO. Repercussões gerenciais da rotatividade 
de pessoal sob a ótica de enfermeiros: pesquisa exploratória. Online braz j nurs. [Internet] 2014; 13(4) [acesso 21 jan 2015]. Disponível: http://dx.doi.org/10.5935/1676-4285.20144394.

11. Costa GM, Figueiredo RC, Ribeiro MS. A importância do enfermeiro junto ao PSE nas ações de educação em saúde em uma escola municipal de Gurupi - TO. Revista Cientifica ITPAC. [Internet] 2013; 6(2) [acesso em 22 jul 2014]. Disponível: http://www.itpac.br/arquivos/Revista/62/6.pdf.

12. Leite CT, Vieira RP, Machado CA, Quirino GS, Machado MFAS. Prática de educação em saúde percebida por escolares. Cogitare Enferm. [Internet] 2014; 19(1) [acesso em 21 jan 2015]. Disponível: http://dx.doi.org/10.5380/ ce.v19i1.35925.

13. Rivemales MCC, Souza RG, Souza MKB. Primary Care Information System as a management tool: study case in Santo Antônio de Jesus / BA. Online braz j nurs. [Internet] 2012; 11(1) [acesso em 23 jul 2014]. Disponível: http:// www.objnursing.uff.br/index.php/nursing/article/view/3552.

14. Ministério da Saúde (BR). Secretaria de Atenção à Saúde. Departamento de Atenção Básica. Cadernos de Atenção Básica - Saúde na Escola. Brasília (DF): Ministério da Saúde; 2009.

15. Al-Rowaily MA. Prevalence of refractive errors among pre-school children at King Abdulaziz Medical City, Riyadh, Saudi Arabia. Saudi J Ophthalmol. 2010; 24(2) [acesso em 27 Out 2013]. Disponível: http://www.ncbi.nlm. nih.gov/pmc/articles/PMC3729549/.

16. Teixeira MB, Casanova A, Oliveira CCM, Ensgtrom EM, Bodstein RCA. Avaliação das práticas de promoção da saúde: um olhar das equipes participantes do Programa Nacional de Melhoria do Acesso e da Qualidade da Atenção Básica. Saúde debate. [Internet] 2014; 38(n.esp) [acesso em 02 fev 2015]. Disponível: http://dx.doi. org/10.5935/0103-1104.2014S005.

17. Clancy A, Leahy-Warren P, Day MR, Mulcahy H. Primary Health Care: Comparing Public Health Nursing Models in Ireland and Norway. Nurs Res Pract. [Internet] 2013; (2013) [acesso em 29 set 2013]. Disponível: http:// dx.doi.org/10.1155/2013/426107. 